\title{
Formación de la identidad docente de estudiantes de la carrera de Educación Primaria desde la experiencia de la práctica profesional
}

\author{
María L. Madueño* y Lorena Márquez \\ Instituto Tecnológico de Sonora, Departamento de Educación, 5 de Febrero 818 Sur, Col. Centro, Ciudad Obregón, \\ Sonora, México (correo-e: maria.madueno@itson.edu.mx).
}

* Autor a quien debe ser dirigida la correspondencia

Recibido Dic. 24, 2019; Aceptado Feb. 28, 2020; Versión final Abr. 29, 2020, Publicado Oct. 2020

\begin{abstract}
Resumen
El propósito de este estudio fue comprender qué cualidades de la práctica profesional favorecen la formación de la identidad docente de estudiantes de la carrera de educación primaria. La investigación fue cualitativa. La información se recuperó mediante grupos focales con la participación de 15 estudiantes de una institución de educación superior española. A partir de los resultados se destaca que la práctica profesional favorece el desarrollo de la identidad docente desde funciones relacionadas con la observación, apoyo e impartición de clases. Dichas funciones cobran significado cuando el estudiante se involucra en la acción docente y reflexiona sobre la práctica desarrollada. Se concluye que las prácticas profesionales constituyen una importante ruta para conformar la identidad docente durante la formación inicial del futuro profesor, para ello es necesario involucrarse en el ejercicio docente y asumir la identidad como un proceso dinámico que se construye desde la experiencia.
\end{abstract}

Palabras clave: identidad docente; formación docente; práctica profesional; estudiantes de educación

\section{Formation of teaching identity of students of the career Primary School Pedagogy from the experience of the professional practice}

\begin{abstract}
The purpose of this research study was to understand what qualities of professional practice favor the development of teaching identity in students of the career Pedagogy in Primary School. The research was qualitative and the information was recovered through focus groups with the participation of 15 students from a Spanish higher education institution. The results showed that professional practice favored the development of teaching identity from functions related to observation, support, and teaching. These functions acquire their real meanings when the student engages in teaching action and reflects on the practice developed. It is concluded that professional practices constitute an important route to shape the teaching identity during the initial training of the future teacher. To achieve this, it is necessary to get involved in the teaching exercise and to assume identity as a dynamic process that is built from experience.
\end{abstract}




\section{INTRODUCCIÓN}

En el ámbito educativo, la docencia se asocia con el incremento de expectativas sociales de la infancia y, en ese sentido es un tema de interés (Organización de las Naciones Unidas para la Educación, la Ciencia y la Cultura UNESCO, 2016). La docencia, entendida como una labor compleja, requiere que las instituciones educativas promuevan condiciones y acciones idóneas para una formación de la identidad docente durante la trayectoria formativa de los estudiantes. La práctica profesional representa una de las principales estrategias para fortalecer la formación del estudiante en las instituciones de educación superior (Zabalza, 2016), y de igual manera, dicha práctica incide en la formación identitaria del futuro profesionista (Corbella y Alonso, 2019); es por ello que el interés de la presente investigación se centra en el estudio de la práctica con relación a la identidad docente.

La preocupación por quien se desempeña en la docencia requiere prestar atención a su identidad, lo anterior es necesario si se considera que la identidad es un referente de actuación, debido a que el ser humano actúa a partir de lo que es (Bruner, 1991). A partir de Bruner (1991) resulta interesante indagar las particularidades del proceso en el que se va conformando la identidad; es decir, es importante estudiar la configuración docente, desde los primeros años de preparación profesional de quienes se forman como profesores, dado que ello se relacionará, en un futuro, con un desempeño profesional exitoso reflejado en una mejor educación para sus alumnos, independientemente del nivel educativo del que se trate (Robinson, et al., 2018).

Arvaja (2016) señala que la identidad docente se encuentra asociada con la biografía individual y con el desarrollo del ambiente social. La construcción de la identidad inicia previo a los procesos de formación inicial, y de igual forma, la experiencia vivida como estudiante es parte de dicha construcción. Para Cantón y Tardif (2018) definir el concepto de identidad docente es un asunto complejo, se considera un proceso que se da al interior de las personas gracias a sus experiencias de vida personales y profesionales, por lo cual, se construye y reconstruye desde la propia práctica docente conforme al tiempo, espacio e interacción con otros. Es importante destacar que la escuela forma parte del contexto sociocultural de la construcción de identidad por lo que incide en su definición (Cortés, et al., 2014), es decir, las oportunidades formativas gestadas en las instituciones formadoras de futuros formadores son detonantes importantes en el proceso de la construcción de la identidad docente. Cabe señalar que la identidad docente se concibe como la definición que el docente hace de sí mismo, y a la vez, del grupo profesional al que pertenece; es una concepción que se extiende desde y hacia sus experiencias personales y sociales en el contexto específico en que se desarrolla.

Muñoz y Arvayo (2015) afirman que la formación de la identidad docente requiere de un proceso de construcción dinámico, a partir de interacciones sociales y experiencias en el área de desempeño. Dada la importancia de la identidad y el reconocimiento de su construcción en el área de desempeño profesional, Corbella y Alonso (2019) refieren las prácticas profesionales como una herramienta institucional idónea de formación, lo anterior, al sostener que el estudiante tiene la ventaja de estar en contacto con el contexto real de su campo de acción, y porque de esa manera, poco a poco adquiere una nueva forma desde contextos reales que le permiten resolver problemas y proponer soluciones. En sí misma, la práctica profesional se advierte como una estrategia de influencia en el curriculum (Zabalza 2016), y más aún, ante las críticas hacia la formación universitaria, mismas que exigen ser consistentes con las realidades del contexto social y mayor compromiso con la formación práctica de los futuros profesionistas.

La práctica profesional, como curso formativo para los futuros docentes, es una oportunidad para tejer la teoría aprendida con su práctica, lo anterior, a través de la acción-reflexión-acción y de las interacciones sociales con los diferentes actores educativos; y a su vez, dicha práctica e interacción favorecen el ser docente al conjugarse como experiencias movilizadoras de conocimientos (Palazzo, et al., 2016). A partir de lo anterior, se enfatiza la importancia de los escenarios de práctica profesional porque se potencia la idea de que los estudiantes transciendan la formación áulica recibida en la universidad, para preponderar la preparación en contextos situados que contribuyan a su formación como docentes (Zabalza, 2016).

La relevancia de la identidad se debe a que facilita el ejercicio de una ocupación laboral de forma integral, con calidad y compromiso, permitiéndole, a quien la posee, reconocerse y ser reconocido como un integrante de un gremio profesional (Muñoz y Arvayo, 2015) y esto incluye a quien funge como docente. Las instituciones educativas, que forman al profesorado de educación primaria, necesariamente deben atender rutas hacia la formación de la identidad docente de sus estudiantes, en este sentido, la práctica profesional es uno de los caminos para construirla y para fortalecerla. De acuerdo con Vanegas y Fuantealba (2019), el proceso de identidad es un reto para los futuros profesionales de la educación porque implica cambios en conocimientos, emociones y responsabilidades, cabe señalar que lo anterior puede ser confuso o incierto 
durante la formación inicial docente, sin embargo, es algo que se fortalece durante el proceso de formación, por lo que una buena experiencia de práctica profesional suele ser una estrategia necesaria para forjar identidad y para dar paso a identificarse como el profesor. Además, es importante destacar que, dicha práctica involucra al estudiante en contextos de aprendizajes situados posibilitando la adquisición de conocimientos, habilidades y competencias necesarias para el ejercicio profesional (Chan-Pavón et al., 2018). Por ello, la práctica profesional en la formación inicial de estudiantes de educación primaria debe cuidarse para favorecer la calidad formativa de quienes forman a otros, y en particular a la infancia.

Robinson et al. (2018) identificaron que la construcción de la identidad profesional se construye desde tres dimensiones: personal, formación inicial y ejercicio profesional; además, subrayaron que la vinculación con la experiencia diaria, así como la relevancia de la valoración positiva que los demás emiten de su actuación profesional; también señalan que es crucial profundizar y conectar el tema de formación inicial e identidad a partir del desarrollo de competencias profesionales en donde se potencien posturas críticas y actuales para abordar la complejidad del acto educativo en escenarios diversos e incidir a nivel de políticas públicas con la intención de favorecer la calidad de la educación infantil.

Uno de los rasgos centrales del desarrollo de la identidad profesional es que se sitúa en un tramo significativo de la vida personal, académica y laboral, constituyendo un proceso en continua recomposición (Jarauta y Pérez, 2017). Las identidades profesionales se configuran a partir de un proceso de socialización, en conjunción con otras personas, en espacios profesionales de relación en los que, mediante identificaciones, representaciones y atribuciones desarrolladas en el transcurso del tiempo e interpretadas de acuerdo a las situaciones vividas por el sujeto y desde sus relaciones con otros (Jara y Mayor-Ruíz, 2019)

Es importante destacar que, si la calidad educativa tiene como uno de sus pilares la actuación del docente, será necesario ampliar estudios en torno a dicho actor, Jarauta y Pérez (2017), enfatizan que, al situar la mirada en el docente de educación inicial o básica, se requiere estudiar cómo conforma su identidad como docente. Desde otros estudios se ha reportado el proceso de construcción de la identidad docente y profesional; algunos señalan reflexiones o tipologías para referir momentos, personajes o dimensiones (Pearce y Morrison, 2011; Pérez y Quijano, 2018) que ayudan a la comprensión de su formación. otros estudios reportan hallazgos a sobre rutas de formación que favorecen dicha construcción; sin embargo, se requiere seguir profundizando a partir de la recuperación de la experiencia y significados que estudiantes de una licenciatura en educación primaria, otorgan a la práctica profesional como un elemento articulador del ser docente como futuros profesores, y es precisamente dilucidar el impacto de esos primeros contactos con la profesión lo que esta investigación se ha propuesto para contribuir al entendimiento de ese proceso de construcción y abonar en la atención de su identidad profesional.

Además de lo citado con anterioridad, desde un estudio de tipo cualitativo, desarrollado con estudiantes de educación de la Universidad de Barcelona, se reportó que la construcción de la identidad como maestros, está influida tanto por experiencias previas, motivación personal y a partir de la trayectoria que se adquiere como estudiante al vivir la realidad como futuro maestro, sin anclarse a una perspectiva como alumno; y desde dichos hallazgos cobra significado la práctica profesional como escenario de aprendizaje porque se implementan ideas, expectativas y preconcepciones que ayudan a contrastar lo ideal con lo real (Pillen, et al., 2012; Jarauta y Pérez, 2017). La experiencia desde la práctica, y la posibilidad del contraste entre realidad e ideal, está asociada con la reflexión teoría práctica (Sutherland, et al., 2010; Vaillant y Marcelo, 2015; Cantón y Tardif, 2018).

La experiencia de la práctica profesional, aunada a la reflexión y al significado de dicha práctica por parte del estudiante, fortalece la identidad y la formación docente; al respecto, García, et al. (2016) mencionan que la práctica profesional, de los estudiantes de educación, al ser el primer contacto con la realidad laboral, fortalece su identidad profesional. En consideración a estas ideas se considera importante explorar la siguiente pregunta de investigación, desde la perspectiva de los estudiantes: ¿Qué cualidades de la práctica profesional favorecen la formación de la identidad docente, a partir de su experiencia? El propósito del estudio se orientó a comprender qué cualidades de la práctica profesional favorecen la formación de la identidad docente de estudiantes de educación primaria.

\section{MÉTODO}

Se optó por un abordaje desde lo cualitativo, dada la pretensión de esta investigación por acercarse al estudiante y futuro profesional de la educación, para recuperar desde su propia voz las experiencias y significados en torno a la realización de las prácticas profesionales y el rol que toman en su proceso como cualidades que contribuyen a identidad docente (Denzin y Lincoln; 2012; Schettini y Cortazzo, 2015; Rapley, 2018). 


\section{Participantes}

La investigación se desarrolló con estudiantes de Licenciatura en Educación Primaria de una universidad pública de Madrid, España. Para la elección de los participantes se empleó un muestreo selectivo dado que se debían cubrir determinadas características conforme al propósito del estudio: ser estudiante inscrito en un programa educativo afín a la educación, haber cursado y acreditado (aprobado) dos cursos de práctica profesional (definida en el currículum o plan de estudio), aceptar compartir su experiencia en la modalidad de entrevista grupal y ser grabado en audio y video.

Participó un total de 15 estudiantes con edades entre los 20 y 24 años, los cuales fueron distribuidos en dos grupos focales según la disponibilidad de sus horarios de clase. El primer grupo focal se confirmó por siete estudiantes, de las cuales el $100 \%$ son mujeres y el grupo 2 se constituyó por dos hombres y seis mujeres. Todos practicaron en educación primaria, solo una estudiante del grupo 2 realizó la práctica en institución privada y los demás en colegios públicos. La identificación de los participantes se ilustra con una homoclave compuesta por un número que los identifica seguida de un guion con el número asignado a cada grupo focal; por ejemplo, E1_1 representa a la estudiante 1 del grupo focal 1 y E1_2 representa al estudiante 1 del grupo focal 2.

\section{Técnica e Instrumento}

La recuperación de información fue con la técnica de grupo focal con la finalidad de promover la interacción entre los participantes, desde la experiencia de práctica. De acuerdo con Then et al., (2014), entre las ventajas de los grupos focales se encuentra la oportunidad de explorar en el significado y la experiencia de personas que comparten intereses o vivencias, así como la opción de recuperar datos de forma ágil y potenciando el cruce de opiniones; para ello, éstos autores sugieren integrar grupos de cuatro a 14 integrantes.

El instrumento empleado fue una guía de entrevista, misma que se validó con el apoyo de pares. La estructura del instrumento consideró tres momentos. Primero, la obtención de datos de identificación, se solicitó a cada participante: su nombre real o ficticio para nombrarlos durante la sesión; su edad; especificar los lugares o escenarios donde realizaron la práctica, definiendo el semestre en el que se realizó y la duración de la misma y, por último, mencionar la función desarrollada por cada periodo. En el segundo se plantearon las siguientes preguntas: ¿Qué te forma como docente?, ¿Cómo contribuye la práctica en el proceso de formarte como docente?, a partir de la práctica, ¿Qué te ayuda a sentirte docente? y, ¿Qué significó para ti la práctica en los escenarios? El último momento consistió en el cierre de la sesión con un espacio de tiempo para solicitar a los participantes agregar algo adicional.

\section{Procedimiento}

El trabajo de campo se desarrolló en noviembre de 2018 durante una estancia de investigación y el proyecto concluyó su desarrollo en mayo de 2019. Para el acceso al campo se solicitó autorización a las autoridades académicas correspondientes. Como porteros fungieron dos profesores de la Universidad, ello facilitó identificar y contactar a los estudiantes participantes.

Al invitar a los estudiantes a participar se proporcionó a cada uno la carta de consentimiento informado que detallaba el propósito de la investigación, el procedimiento de recuperación de información, incluido el uso de equipos de audio y video, así como el manejo, resguardo y uso que se dará a la información proporcionada, haciéndose énfasis en la confidencialidad y anonimato. Los estudiantes que expresaron su interés en participar al firmar el consentimiento y eligieron entre dos horarios definidos para los grupos focales. Cada grupo focal se desarrolló en días distintos, con un tiempo aproximado de 45 minutos, en un aula pequeña y libre de distractores proporcionada por la universidad en días diferentes, las investigadoras desarrollaron las sesiones desde el rol de coordinadora y moderadora.

Los datos fueron procesados mediante análisis de contenido a través de un proceso de categorizaciónanálisis-interpretación. Un primer paso fue realizar las transcripciones fieles de los audios-videos obtenidos, al transcribir se cuidó asignar una homoclave a cada participante para identificarlo durante el análisis y para citar sus narrativas como evidencia empírica. Como apoyo para el proceso de análisis se empleó el programa Atlas.ti, después de cotejar las transcripciones se ingresaron al software para proceder a la lectura y relectura de las mismas hasta lograr la familiarización e identificación de palabras o frases significativas para el estudio, acto seguido se marcaron las citas relevantes y se etiquetaron con códigos, estos últimos se organizan y agrupan para hacer emerger las categorías. Posteriormente se organizó la información por categorías identificando y seleccionando citas desde el Atlas.ti como expresiones significativas de los participantes, las cuales se presentan como evidencia empírica del tejido narrativo como 
parte del análisis e interpretación de los datos. Como criterio de credibilidad se revisaron y cotejaron las trascripciones, así como la selección de citas y códigos para derivar las categorías, de igual manera se cuidó constatar el cruce de información (Atkins y Wallace, 2012) a partir de recurrencias entre los participantes de los dos grupos focales; como criterio de confiabilidad, se cuidó el anonimato de los informantes mediante el uso de una homoclave compuesta por la letra $E$ (de estudiante), un número consecutivo como diferenciador de cada participante y al final un número seguido de un guion bajo para referir a cada grupo focal.

\section{RESULTADOS}

Los participantes refirieron experiencias derivadas de dos periodos de práctica profesional llevados a cabo en diferentes escuelas primarias, con una duración de tres semanas por periodo. Entre las cualidades, de la práctica profesional, que favorecen la identidad docente destaca: el involucramiento en acciones docentes y la disposición del estudiante hacia el aprendizaje desde la reflexión. Asociado a estas cualidades, a partir del análisis, se identifican condiciones necesarias o idóneas, sintetizadas en la descripción de las siguientes categorías: Involucramiento en la acción docente y reflexión de la práctica.

\section{Involucramiento en la acción docente}

Un atributo o cualidad de una práctica, que incide en la identidad docente, es la inmersión en el aula de clase; es decir, la posibilidad de vivir experiencias con el apoyo de los docentes que están frente a grupo y practicando con los niños. En estos casos, la apertura de los docentes en los escenarios de práctica, para permitirles a los estudiantes experimentar funciones asociadas con la docencia es sustantiva. En las prácticas profesionales, los participantes llevaron a cabo las siguientes acciones: observador en clases, maestro de apoyo en clase y maestro frente a grupo. Desde estas tres funciones se describe la presente categoría, y al mismo tiempo se citan las acciones que cobran significado en torno a la formación de la identidad en tanto docente

\section{Función como observador en clase}

Los entrevistados coinciden en que, por lo general, durante los primeros periodos de práctica su función es de observador en un aula de clase en primaria, y son asignados a un profesor o profesora que orienta y supervisa su desempeño. Cuando se funge como observadores, la acción cotidiana desarrollada por los profesores titulares de grupo, es referida como un aprendizaje que contribuye a la formación identitaria; sin embargo, la práctica de observación cobra mayor riqueza cuando se experimenta la opción de observar a diferentes docentes en el periodo de práctica.

En los dos grupos se coincidió que es sustantivo observar cómo trabaja la maestra o maestro" (E7_2, E2_1, E6_1, E6_2, E7_1). Por otra parte, se dijo que "se aprende más en las prácticas viendo a otros profesores trabajar, que con la teoría que nos puedan dar en las clases de la universidad" (E7_1). E6_1 refirió: "a mí me ayuda personalmente el modelo del profesor [su forma de impartir la clase], verlo a la cara, por ejemplo, en las prácticas ves a un tipo de profesor, y vez otro tipo de profesor" lo cual representa mayores oportunidades para cuando alguien requiera ser contratado como docente, en ésta última idea coinciden otros dos participantes del mismo grupo focal y dos del segundo grupo.

Los participantes refieren que la observación es una función básica para ellos como futuros docentes, ya que les permite descubrir cómo es la práctica docente de un maestro de primaria, y eso favorece la construcción de su identidad como docentes. La posibilidad de observar a diferentes profesores y las acciones que forman parte de su modelo de trabajo representa un referente para sus prácticas futuras.

La opción de observar a diferentes profesores les permite conocer cómo llevan a cabo la docencia a partir de la asignatura que imparten, por ejemplo la participante E6_2 dijo: "se me permitió moverme por otras aulas con otros profesores y también en un centro bilingüe, la tutora solo daba asignatura de matemáticas en castellano, por lo cual se me permitió ver cómo es la educación bilingüe" y eso, reitera la participante, representa una ayuda importante en el proceso de identificarse como docente.

Por su parte, E2_1 destaca: "la profesora de música me dejaba entrar con ella en las aulas para ver cómo eran las clases", en este caso, la estudiante resalta que la experiencia de observar tanto a la maestra de su grupo como a la de música le ayudó a ella a ampliar su proceso de aprendizaje en torno a la función docente. Ampliar la oportunidad de contextos de observación, en un mismo periodo de práctica constituye una cualidad necesaria para favorecer el proceso de formación como docente de los estudiantes de educación primaria, sobre todo si los docentes son de diversas áreas o especialidades. 


\section{Función como maestro de apoyo en clase:}

Los participantes E1_2, E2_1, E2_2, E3_1, E3_2, E4_2, E5_2, E6_1, E6_2, E7_2 y E8_2 expresaron que es mejor cuando se interviene más durante la práctica profesional. Refirió que la función como maestro de apoyo en clase es una oportunidad de actuación para trabajar con niños que requieren ayuda extra para lograr los propósitos de aprendizaje, definidos por el maestro titular del grupo. Es importante mencionar que esta oportunidad se da preponderantemente en el segundo periodo de práctica. Cabe señalar que, de acuerdo con lo planteado por los estudiantes, de los dos grupos focales, cuando se les involucra en diversas actividades relacionadas con el apoyo a los alumnos del grupo y en la corrección de exámenes, la función desempeñada cobra mayor significado con relación a la identidad como docente. Lo anterior, debido a que en la medida que ellos actúan en un campo real, inmersos en el abordaje de diferentes necesidades de aprendizaje de los niños, se generan condiciones para poner en práctica los conocimientos teóricos y para implementar estrategias pertinentes para que el alumno aprenda, lo cual ayuda a permear su identidad docente.

Con respecto a lo anterior E1_2 comparte: "en una clase hay muchos niños con muchas coyunturas diferentes y no se les puede dar clase igual [...] es importante saber de teoría de la educación", pero que es igual o más importante esa parte del trabajo docente en el que "para saber enseñar, pues habrá que meterse en harina, mancharse las manos", con ello se destaca la importancia de la práctica en contextos reales y ante situaciones propias del desempeño, en donde la teoría por sí sola no tiene sentido; así también lo expresan otros tres participantes: "Yo también hacia algunas tareas como prestar apoyo a los alumnos en lo que más les costaba, debía estar más al pendiente de ellos" (E3_2), "En el primer año mi tarea consistía prácticamente en apoyo para los alumnos y en ayudar a la profesora en sus tareas como corregir exámenes" (E4_2) y finalmente, E8_2 expresa: "Estaba de apoyo con niños que les costaba más, realizamos un taller de lectura y yo me iba al final de la clase a leer con los niños".

En el primer grupo focal, una participante señaló que debe de existir un involucramiento en el escenario de la práctica profesional, para poder formarse como docente e ir construyendo así su identidad como tal. También se dijo que durante estas prácticas se presentan ciertos obstáculos y que uno de ellos, es cuando el profesor del escenario restringe la participación del practicante solo a observar las clases que imparte. Sin embargo, otros estudiantes señalaron que esa situación depende de la persona que está en formación, porque cuando se muestra interés se puede lograr la apertura del maestro del escenario, a éste respecto se dijo: "A lo mejor tienes a un profesor que no te ayude en nada, pero si tú preguntas...¿ ¿puedo hacer esto, puedo ayudarte en tal?, y estás constantemente intentando hacer tus prácticas, pues también hay un interés en la persona [se refiere al estudiante practicante] de que quiere seguir aprendiendo, eso es lo que más me ha ayudado (E3_1, enfatiza que le funcionó ser persistente con el docente para lograr la oportunidad de involucrarse más y aprender)". El significado atribuido a la práctica, con respecto a la formación e identidad docente, fue referido en términos del involucramiento en las funciones desempeñadas. En síntesis, para los participantes, involucrarse como maestro de apoyo en clase, es clave para formarse como docente, lo cual, fue un aspecto en el que la mayor parte del primer grupo foca coincidió. Además, de acuerdo con lo señalado en ambos grupos, la práctica como maestros de apoyo pone a prueba la vocación, los conocimientos y la capacidad para resolver las necesidades de los niños con quienes se trabaja (E1_2, E3_1, E3_2, E4_2 y E8_2).

\section{Función como maestro frente a grupo}

La función como maestro frente a grupo es más compleja que las anteriores y la de mayor riqueza en el proceso formativo del estudiante de educación, sin embargo, algunos no tienen la oportunidad de experimentarla en sus periodos de práctica. La asignación de la función depende de la confianza y apertura que tenga el profesor del curso para brindarles esa libertad de actuación. Los estudiantes que experimentaron estar frente a grupo, la refieren como la de mayor grado de impacto en su construcción identitaria como docentes porque les ayuda a poner en práctica los conocimientos teóricos adquiridos en la universidad, así lo menciona E1_2 "la práctica es esencial para hacer algo bien, sin quitarle importancia a lo que es la teoría porque para ser profesor pues hay que saber muchas cosas del desarrollo de los niños, desarrollo cognoscitivo", y además agrega que en necesario entender que cada clase y cada niño son diferentes.

También, se logró identificar que estar frente al grupo, dar una clase e intervenir más, permite al estudiante solucionar problemas que, durante su preparación teórica, en aula de la universidad, no adquieren. Al respecto E7_1 mencionó que "en las clases de la universidad sí que se imparten una serie de relatos [o cúmulo] de información, pero cada niño es un mundo y hasta que no trabajas con ellos no sabes cómo solucionar equis cosas o cómo enseñarles". En este sentido, cobra significado la oportunidad de interveniractuar en un contexto real, ya que las acciones que realizan están apegadas a su campo formativo, y a su 
vez, es posible relacionar las acciones con lo aprendido durante su preparación en la universidad, a éste último respecto, la participante E3_1 compartió: la profesora del escenario de práctica "me dejaba marcharme con los niños [salir del aula con los alumnos] que necesitaban alguna cosa [apoyo relacionado con la clase], ya sea de matemáticas o de lengua" para esta participante, así como para E2_2, E3_2, E8_2 la oportunidad de actuación durante la práctica profesional es esencial en su ser docente. Con relación a lo anterior, la libertad y confianza brindada al practicante, es una condición necesaria en el proceso formativo, lo cual, tiene la ventaja de aplicar lo aprendido en contexto, lo cual, desde la voz de los participantes del estudio, les ayuda a descubrir nuevos aprendizajes o reforzar lo que ya se sabe.

Los estudiantes que participaron en la investigación hacen hincapié en que la práctica profesional, en escenarios reales, favorece su identidad docente, sobre todo al participar en funciones relacionadas con observar la clase de un profesor, apoyar en las diferentes actividades académicas de los niños, y sobre todo desde la experiencia de estar frente a grupo impartiendo clases, lo anterior porque relacionan la teoría con la práctica e identifican los métodos de enseñanza más acorde a su estilo, teniendo la oportunidad de reflexionar re la práctica docente que en un futuro desean o no aplicar. Estas ideas son acordes a lo planteado por, Cortés et al. (2014), quienes señalan que el contexto sociocultural de la escuela, como escenario de práctica, es un elemento que influye en la conformación de la identidad docente de este actor educativo por la riqueza de interacciones sociales que éste provee.

La identidad docente, de los estudiantes de primaria, se afianza a partir de la percepción que se forman de la función docente, sobre todo ante la posibilidad de involucrarse en dicha práctica, en cada una de las funciones antes referidas en este apartado, lo anterior, al experimentar diversas actividades relacionadas con en el contexto áulico. En este caso, se coincide con lo planteado por Bolívar et al. (2014) y por Muñoz y Arvayo (2015) quienes destacan la importancia de la interacción social y la experiencia en contextos reales apegados a lo que experimentarán en su futuro desempeño profesional.

\section{Reflexión de la práctica}

Una característica deseable desde la práctica profesional es la reflexión. Los alumnos afianzan el ser docentes desde el ejercicio de la docencia, cuando realmente se implican en ella; también se identificó que para los participantes cobra significado el aprendizaje que surge a partir de los momentos en los que comparten experiencias de práctica con sus compañeros. Las reflexiones se relacionan con los momentos de preparación para la práctica y posteriores a ella, así como al compartir experiencias y materiales con sus compañeros. Se gestan aprendizajes desde sí mismos y desde otros.

Los participantes E3_1, E3_2, E4_1, E5_2, E6_2 indican que aprenden de sí mismos cuando se preparan para desarrollar una práctica profesional idónea, porque aun cuando en su formación universitaria los proveen de los fundamentos teóricos para su desempeño docente, identifican que tienen la necesidad de preparase más para ejercer su periodo de práctica (principalmente, el segundo), sobre todo en cómo van a facilitar los contenidos de aprendizaje a sus alumnos. La participante E6_2 refiere "todas las asignaturas se llaman fundamentos y didáctica de... pero yo creo que siempre nos quedamos en la parte de fundamentos, y la didáctica la dejamos olvidada". E3_1, E3_2, E4_1, E5_2, coinciden que la didáctica no debe dejarse de lado por la teoría, cuando mencionan que los profesores de la universidad se centran más en la parte de los fundamentos.

En este sentido, los practicantes buscan información sobre cómo ayudar a que el estudiante aprenda el contenido, por lo que, se documentan sobre aspectos didácticos que beneficien su quehacer docente, y así poder elaborar materiales con la finalidad de ayudar a los niños aprender. El proceso de búsqueda, documentación y elaboración de materiales didácticos permite que los participantes reflexionen sobre lo que están haciendo y así aprenden de sí mismo, siendo autodidactas y proactivos en su formación docente. En estos casos, la reflexión a partir de lo que deben hacer de forma previa, así como identificar y reconocer que requieren ampliar sus saberes, es parte de su proceso de formación como docentes, que a su vez les ayuda a identificarse como tales. Esta ruta de aproximación en el ser docente, desde lo expuesto por Vaillant y Marcelo (2015) favorece el contraste de la experiencia asociado con la reflexión de la actuación.

De igual manera, los participantes E1_1, E2_1, E3_1, E8_2 indican que aprenden de sí mismos cuando generan una reflexión que los lleva a diferenciar qué hacer y qué no hacer durante la práctica profesional, lo cual, a su vez les permite identificar prácticas docentes buenas y malas, misma que los conducen a reflexionar sobre aquello que se debe o no realizar como maestro. Una participante menciona: "Yo, en mi caso, he visto a dos profesores, y sé lo que quiero hacer y lo que no quiero hacer en mi futuro, he visto cosas que no me han gustado, pero tampoco puedes decir mucho de si esto está bien o no. Cuando tú tienes un profesor [se refiere al maestro del escenario de práctica] que te puede guiar, y que te dice "esto está bien, esto no", creo que ahí es donde de verdad se aprende (E3_1)". Aquí la figura del profesor guía 
logra trascender en la formación inicial del futuro docente; de acuerdo con Izadinia (2016) bajo una adecuada tutela y orientación no solo se refuerza la identidad como docente, sino también la confianza y seguridad del estudiante practicante. Este acompañamiento resulta crucial en el ser docente durante la formación inicial.

La reflexión diferenciadora entre lo que sí y lo que no se debe realizar, lleva al practicante a reconocer que esta cualidad de la práctica profesional cobra valor para la formación de su identidad docente. Al respecto una estudiante refiere: "He sentido que quizá me ha faltado tiempo en las prácticas porque llega un momento, con esas tres semanas, de que estas tan a gusto, y dices qué pena no poder seguir, y llegué a sentir que había aprendido más en esas tres semanas que lo que llevo en dos años de carrera (E1_1)". En ese sentido, a su decir se reconoce la necesidad de disponer de un mayor tiempo en el escenario para seguir aprendiendo, lo anterior a razón de que, para ellos este periodo de inmersión en un contexto real favorece aprender de sí mismo e identificarse como docentes.

Una de las cualidades de la práctica profesional es que permite aprender de otros. Los participantes E1_1, E4_1, E6_1, E4_2 refieren aprender de sus compañeros pares. A manera de ejemplo se ilustra lo expresado por E1_1: "Durante la carrera nos han mandado al trabajo en equipo y más que trabajo en grupo es ver las ideas de otras personas; por ejemplo, nuestro grupo ha planteado esta forma de ejercicio, estos materiales y herramientas y de repente vez que están haciendo otra cosa distinta [en un equipo-grupo diferente] y que ni te había pasado por la cabeza y que parece buena idea". Además, compartir estrategias y materiales entre compañeros favorece su formación docente porque reconocen lo que están trabajando otros, y a partir de ello, reflexionan de qué manera eso les puede ayudar a mejorar su quehacer. Algo esencial para poder aprender de otros es saber escuchar, poner atención en lo que otros comparten, así lo refiere uno de los entrevistados: "Escuchar a los demás cuando haces un trabajo en grupo, creo que eso también ayuda, por ejemplo, hay que hacer una actividad de no sé qué, a ti te puede salir una idea y a otro compañero otra, y las fusionas y sale algo mejor (E6_1)". El trabajo en equipo es una estrategia importante porque contribuye a que el estudiante se forme e identifique como docente, no obstante, el estudiante debe poseer una mente abierta y darse la oportunidad de aprender de otros compañeros, ya que, si solo escucha y no aplica la mejora, esta estrategia no favorece su formación docente.

La reflexión sobre la práctica profesional que se genera entre compañeros después de experimentar la práctica es muy relevante en el proceso de identificarse como docentes. A manera de ejemplo, un participante dijo: "Hablar con los compañeros, por ejemplo, cuando vuelves de las prácticas, lo comentas con tus compañeros que están en otros colegios y han tenido otras realidades y creo que eso ayuda mucho, tanto para verlo globalmente, como para ver lo que tú has vivido desde otro punto de vista (E7_2)". Es decir, los encuentros posteriores a los periodos de la práctica profesional son deseables para los participantes porque aun cuando no pudieron estar en otros contextos, desde la socialización de experiencias con sus compañeros, viven y reconocen lo que otros vivieron, dicha reflexión entre pares contribuye a irse conformando como docentes. Se insiste en la relevancia que cobra la interacción social con los compañeros, tanto antes como después de la participación en los escenarios (Bolívar et al, 2014; Muñoz y Arvayo, 2015), Desde estas ideas, puede decirse que compartir la experiencia de lo que se hace en los escenarios de práctica, seguida de procesos reflexivos, representa un aliado de la construcción identititaria, y además constituye un aprendizaje para el ejercicio profesional.

Para los participantes, ser un buen profesor cobra un significado que los moviliza a aprender. Ellos manifiestan su compromiso de mejorar su quehacer porque consideran que es delicado trabajar con niños que inician una educación formal, asumen que su papel como docentes influye en el camino formativo de los niños que están o estarán a su cargo. Requieren ser buenos docentes porque su ejemplo, dedicación y esfuerzo será un modelo para los niños, en esto coincidieron todos los participantes. E51 dijo al respecto: "Ser profesor es un papel súper importante (...) el papel que tú tienes con los niños les va a marcar su futuro, pasan por lo menos seis horas contigo como profesor y, si tú no eres capaz de ayudarles a seguir una trayectoria, en su camino puedes marcar, puede ser un papel fundamental para ese niño".

Los participantes del estudio, como profesores en formación, deben concentrarse en aprender a ser docentes empáticos con sus alumnos y no centrar la atención en el contenido solamente. Hacen notar que el aspecto socioemocional de los niños es un área que no se puede dejar de lado, al respecto E3_1 menciona que es necesario el "esfuerzo y dedicación por y para los niños, porque se está muchas horas con ellos" y otros agregan: "Pasas mucho tiempo con ellos y no solo les enseñas asignaturas de la vida, como lenguas, matemáticas, sino a saber ser personas, a saber expresar sus sentimientos, sus emociones y eso no solo te vale por las horas activas en el cole, es trabajo de cómo enseñar al niño a cómo hacerlo (E4_1; E5_1)". En resumen, las principales cualidades o condiciones necesarias de una práctica profesional que favorecen la identidad docente, de los estudiantes de educación primaria se presentan en la tabla 1. 
Tabla1. Cualidades de la práctica profesional desde la perspectiva del estudiante

\begin{tabular}{|c|c|}
\hline Categorías & Características de la cualidad identificada \\
\hline $\begin{array}{l}\text { Involucramiento en la } \\
\text { acción docente }\end{array}$ & El involucramiento refiere una práctica que: \\
\hline Como observador & $\begin{array}{l}\text { - } \quad \text { Posibilita observar cómo imparten clase diferentes docentes } \\
\text { - } \quad \text { Es un referente de actuación }\end{array}$ \\
\hline $\begin{array}{l}\text { Como maestro de apoyo } \\
\text { en clase }\end{array}$ & $\begin{array}{ll} & \text { Permite la inmersión en el aula } \\
\text { - } & \text { Favorece trabajar con niños con diversas necesidades } \\
\text { - } & \text { Impulsa el desarrollo de diferentes actividades docentes } \\
\text { - } & \text { Sitúa al estudiante en un contexto próximo a la actuación futura } \\
\text { - } & \text { Cuenta con docentes (en escenario): empáticos, que inspiran confianza y brindan } \\
& \text { oportunidades de aprendizaje. }\end{array}$ \\
\hline $\begin{array}{l}\text { Como maestro frente a } \\
\text { grupo }\end{array}$ & $\begin{array}{ll} & \text { Además de lo anterior... } \\
\text { - } & \text { Permite intervenir y aplicar la teoría. } \\
\text { - } & \text { Ayuda a dimensionar la diferencia entre teoría y práctica } \\
\text { - } \quad \text { Favorece la solución de problemas }\end{array}$ \\
\hline Reflexión de la práctica & $\begin{array}{ll}\text { La reflexión de la práctica profesional requiere (del estudiante): } \\
\text { - } \quad \text { Inmersión en un contexto próximo a la docencia } \\
\text { - } \quad \text { Disposición para aprender de la propia práctica } \\
\text { - } & \text { Socializar ideas entre pares y aprender de ello } \\
\text { - } & \text { Disposición para contrastar la experiencia } \\
- & \text { Compromiso hacia la docencia y hacia los alumnos } \\
\text { - } & \text { Iniciativa y disposición al encuentro de nuevos aprendizajes. }\end{array}$ \\
\hline
\end{tabular}

\section{DISCUSIÓN}

Atender el propósito de precisar las cualidades de la práctica profesional que favorecen la formación de la identidad docente, de estudiantes de educación primaria, puede tomar diversas connotaciones; sin embargo, desde los resultados de esta investigación, se presentan las principales consideraciones, que parten de la experiencia estudiantil, y se ponen en relación con otros hallazgos y reflexiones de estudios previos.

El involucramiento en la práctica, es decir, estar inmersos en el aula y vivir la docencia, ayuda al estudiante a verse a sí mismo como docente. Con base a dicha idea, es necesario recalcar la importancia de lo expuesto por Muñoz y Arvayo (2015) y por Chan-Pavón et al. (2018) quienes señalan que la identidad se gesta en el área de desempeño. De igual manera, es preciso considerar que, de acuerdo con diversos autores como Pillen, et al. (2012), Vaillant y Marcelo (2015), Jarauta y Pérez (2017) y Corbella y Alonso (2019), el campo de acción de un profesional, se afianza desde el contexto real porque es ahí donde se afrontan los problemas reales. De esta manera, la práctica se torna significativa como escenario de aprendizaje, sobre todo cuando los estudiantes pueden intervenir o involucrarse en funciones que les permiten experimentar el contraste entre lo ideal y lo real. Otra cualidad indispensable es la reflexión sobre dicha práctica, con relación a esto último, Cantón y Tardif (2018) visualizan la reflexión como atributo de formación; de igual forma, esta idea se sostiene en Vaillant y Marcelo (2015). En suma, cuando predomina el involucramiento en la acción docente y la reflexión como cualidades clave de la práctica profesional, se favorece la formación del ser docente.

Para comprender cómo es que la práctica está relacionada con la identidad, es oportuno citar los estudios reportados por Cantón y Tardif (2018) y por Muñoz y Arvayo (2015), desde estos autores y lo que aporta la presente investigación, se logra advertir que existe un nexo que es preciso destacar para comprender la relación entre la práctica profesional y el desarrollo de la identidad. Ambos se relacionan a partir de las cualidades formativas desde la acción, que son potenciadas por la práctica y por la naturaleza social desde la que se forma la identidad. Para los autores referidos y desde los hallazgos de este estudio, la formación de la identidad es un proceso que se da con el tiempo a partir de la experiencia, y además, cuando se habla de formación inicial, esa experiencia docente se vive, en gran medida, en los escenarios reales en los que los estudiantes practican. Además, otro nexo importante es la cualidad social de la práctica, ya que en ella prevalecen procesos de interacción, principalmente con los niños con quienes se vive la función docente, y con los docentes del centro que los recibe, y es desde esas interacciones que se enriquece el proceso de formación de los estudiantes. En este sentido, se logra advertir que la interacción constante, entre los actores educativos es una cualidad deseable en una práctica que da forma docente al estudiante y futuro profesionista. 
Por último, se logra advertir que la práctica profesional requiere condiciones idóneas para aspirar a que, a partir de ella, se forje la identidad docente de los estudiantes de educación primaria. La preocupación por cuidar las posibilidades de ampliar el involucramiento de los estudiantes en las funciones que se les asignan en los escenarios, es un elemento clave para mejorar las oportunidades de ofrecer una formación inicial con un sello distintivo de identidad. Y de esa manera, a decir de Vanegas y Fuantealba (2019), se forjarán ricas experiencias; además de que se imprimirá un sello de calidad y compromiso en el desempeño futuro de los estudiantes, esto último de acuerdo con Muñoz y Arvayo (2015). Finalmente, se sostiene que es necesario decidirse y velar por una práctica rica en experiencias, y a su vez, forjar la identidad de quien está a cargo de la formación de otros, porque como lo señala Bruner (1991), quien no se asume como docente, difícilmente actuará como tal.

\section{CONCLUSIONES}

De los resultados de la investigación, de su análisis y discusión, se pueden obtener las siguientes conclusiones: 1) Construirse o reconstruirse como docente requiere, necesariamente, involucrarse en el ejercicio de la labor profesional a la que aspira a ser parte, y en este caso es la docencia. La diversidad de funciones practicadas nutre la idoneidad del desempeño futuro; 2) La práctica profesional, que contribuye a la formación de identidad docente, tiene la cualidad de incorporar la reflexión sobre la práctica desarrollada, para identificar aciertos y aspectos a mejorar; ese proceso de reflexión se enriquece desde la interacción social; 3) Forjar una identidad, desde la práctica, requiere asumir la identidad como un proceso dinámico que se interpreta o reinterpreta desde lo que se experimenta en contextos específicos; y 4) Las instituciones formadoras del profesorado de educación primaria, requieren integrar en sus procesos de formación inicial un esquema de prácticas profesionales con el propósito explícito de forjar la identidad docente, porque quien no se asume como docente, difícilmente se comprometerá y se dispondrá a desempeñarse con idoneidad en el campo docente.

\section{AGRADECIMIENTOS}

Se agradece al Programa de Fomento y Apoyo a Proyectos de Investigación (PROFAPI 2019) del Instituto Tecnológico de Sonora, el apoyo brindado para el desarrollo del proyecto de investigación reportado.

\section{REFERENCIAS}

Arvaja, M., Building teacher identity through the process of positioning, doi: 10.1016/j.tate.2016.07.024 Teaching and Teacher Education, 59, 392-402 (2016).

Atkins, L., y Wallace, S. (2012). Qualitative Research in Education. Londres: SAGE.

Bolívar, A.; J. Domingo y Pérez, P., Crisis and Reconstruction of Teachers' Professional Identity: The Case of Secondary School Teachers in Spain, doi: 10.2174/1875399X01407010106, The Open Sports Sciences Journal, 7, 106-112 (2014).

Bruner, J., Self-making and world-making, doi: 10.2307/3333092, Journal of Aesthetic Education, 25, 1, $67-78$ (1991).

Cantón, I. y Tardif, M., Identidad profesional docente, Narcea Ediciones, Madrid (2018).

Chan-Pavón. M. V., Mena-Romero D.A., Escalante-Euán J. F., y Rodríguez-Martín, M. D., Contribución de las prácticas profesionales en la formación de los estudiantes de la Facultad de Ingeniería Química de la Universidad Autónoma de Yucatán (México), doi: 10.4067/S0718-500620180001000531, Formación Universitaria, (1), 53-62 (2018)

Corbella, M. y Alonso, R. A., El vínculo universidad-empresa en las prácticas curriculares. Investigación e innovación, doi: http://dx.doi.org/10.18172/con.3742, Contextos Educativos, 23, 7-9 (2019).

Cortés, P., A. Leite, y Rivas, J., Un enfoque narrativo de la identidad profesional en profesorado novel. ISSN: 19898614, Tendencias Pedagógicas, 24, 199-214 (2014).

Denzin, N. y Lincoln, Y., Strategies of Qualitative Inquiry, 4ª edición, 209-252, SAGE Publications, Inc, Los Ángeles (2012).

García, S.M., R. González y Martín, A.M., Influencia de las prácticas en el desarrollo de la identidad profesional de los estudiantes de educación social, doi: 10.7179/PSRI_2016.28.18, Pedagogía Social. Revista Interuniversitaria, 28, 245259 (2016).

Izadinia, M., Preservice teachers' profesional identity development and the role of mentor teachers, doi: 10.1108/IJMCE01-2016-0004, International Journals of Mentoring and Coaching in Education, 5, 2, 127-143 (2016).

Jara, C, R. y Mayor-Ruíz, C., Explorar la Construcción de la Identidad Docente en Profesionales de la Salud: Diseño y Validación de Instrumento, doi: 10.4067/S0718-50062019000100013, Formación Universitaria 12(1), 13-24 (2019)

Jarauta, B. y Pérez, M. J., La construcción de la identidad profesional del maestro de primaria durante su formación inicial. El caso de la Universidad de Barcelona, ISSN: 1138-414X, Profesorado. Revista de Curriculum y Formación del Profesorado, 21, 1, 103-122 (2017). 
Muñoz. F. y Arvayo, K. L., Identidad profesional docente. ¿Qué significa ser profesor?, ISSN: 1857 - 7881 , European Scientific Journal, 11, 32, 97-110 (2015).

Organización de las Naciones Unidas para la Educación, la Ciencia y la Cultura Educación 2030 Declaración de Incheon. Hacia una educación inclusiva y equitativa de calidad y un aprendizaje a lo largo de la vida para todos (acceso: 27 de noviembre de 2019) UNESCO (2016).

Palazzo, J., Gomes, C. A. y Sausa Rêgo, G., La práctica en la formación de educador es en Brasil: currículos fracturados, ISSN: 1575-0965, Revista Electrónica Interuniversitaria de Formación del Profesorado, 19, 2, 45-59 (2016).

Pearce, J., y Morrison, C., Teacher identity and early career resilience: exploring the links, Australian Journal of Teacher Education, 36, 1, 47-59 (2011).

Pérez, M. y R. Quijano, R., Análisis del discurso de los estudiantes de Magisterio sobre la contribución del practicum al desarrollo de su identidad profesional docente, ISSN: 1699-2105, Educatio Siglo XXI, 36, 2, 331-352 (2018).

Pillen, M.; Beijaard, D., y Den Brok, P. Tensions in beginning teachers' professional identity development, accompanying feelings and coping strategies, doi: 10.1080/02619768.2012.696192, European Journal of Teacher Education 36(3), 240260 (2012).

Rapley, T. Doing conversation, discourse and document analysis, $2^{\underline{a}}$ edition, SAGE Publications of London, Washington DC, USA, (2018)

Robinson, M. S., Tejada, J., y Blanch, S., ¿Cómo construyen su identidad las educadoras de párvulos principiantes? Una mirada desde diferentes realidades educativas, doi: 10.4151/07189729, Perspectiva Educacional, 57, 3, 104-130 (2018).

Schettini, P. y Cortazzo, I., Análisis de datos cualitativos en la investigación social. Procedimientos y herramientas para la interpretación de información cualitativa, $1^{\text {a }}$ edición, Universidad Nacional de La Plata, La Plata (2015

Sutherland, L.; Howard, S., y Markauskaite, L., Professional identity creation: Examining the development of beginning preservice teachers' understanding of their work as teachers, doi: 10.1016/j.tate.2009.06.006, Teaching and Teacher Education, 26, 3, 455-465 (2010).

Then, K. L., Rankin, J. M., y All, E., Focus group research: what is it and how can it be used? Canadian Journal of Cardiovascular Nursing, 14(1), 16-22 (2014).

Vanegas, C, y Fuanteaba, A., Identidad profesional docente, reflexión y práctica pedagógica: consideraciones claves para la formación de profesores, Perspectiva Educacional. Formación de Profesores, doi. 10.4151/07189729-Vol.58Iss.1-Art.78058(1),115-138, (2019)

Vaillant, D. y Marcelo, C., El ABC y D de la formación docente, Editorial Narcea, Madrid (2015)

Zabalza, M. El Practicum y las prácticas externas en la formación universitaria, Revista Practicum, ISSN: 2530-4550, $1(1), 1-23(2016)$ 
